\title{
Surgical Hand Antisepsis With Alcohol-Based Hand Rub: Comparison of Effectiveness After 1.5 and 3 Minutes of Application
}

\author{
Walter P. Weber, MD; Stefan Reck, MD; Uschi Neff, RN; Ramon Saccilotto, MD; Marc Dangel, RN, MPH; \\ Manfred L. Rotter, MD; Reno Frei, MD; Daniel Oertli, MD, FACS; Walter R. Marti, MD, FACS; \\ Andreas F. Widmer, MD, MS
}

\begin{abstract}
овјестіVE. Research has shown 1.5 minutes of surgical hand antisepsis with alcohol-based hand rub to be at least as effective under experimental conditions as the 3-minute reference disinfection recommended by European Norm 12791. The aim of the present study was to validate the effectiveness of 1.5 minutes of surgical hand antisepsis in a clinical setting by comparing the effectiveness of 1.5- and 3minute applications of alcohol-based hand rub ( $45 \% \mathrm{vol} / \mathrm{vol} 2$-propanol, $30 \% \mathrm{vol} / \mathrm{vol} 1$-propanol, and $0.2 \%$ mecetronium ethylsulphate).
\end{abstract}

DESIGN. Prospective crossover trial in which each surgeon served as his or her own control, with individual randomization to the 1.5or the 3-minute group during the first part of the trial.

SETting. Basel University Hospital, Switzerland.

PARTICIPANTS. Thirty-two surgeons with different levels of postdoctoral training.

MEтноDS. We measured the bactericidal effectiveness of 1.5 minutes and 3 minutes of surgical hand antisepsis with alcohol-based hand rub by assessing the mean $( \pm S D) \log _{10}$ number of colony-forming units before the application of hand rub (baseline), after the application of hand rub (immediate effect), and after surgery (sustained effect) so as to follow European Norm 12791 as closely as possible.

RESULTS. The immediate mean $( \pm S D) \log _{10}$ reduction in colony-forming units (cfu) was $2.66 \pm 1.13 \log _{10}$ cfu for the 1.5 -minute group and $3.01 \pm 1.06 \log _{10}$ cfu for the 3-minute group $(P=.204)$. Similarly, there was no statistically significant difference between the 2 groups with respect to the sustained effect; the mean $( \pm S D) \log _{10}$ increase in bacterial density during surgery was $1.08 \pm 1.13 \log _{10}$ cfu for the 1.5-minute group and $0.95 \pm 1.27 \log _{10}$ cfu for the 3-minute group $(P=.708)$. No adverse effects were recorded.

CONCLUSION. In this clinical trial, surgical hand antisepsis with alcohol-based hand rub resulted in a similar bacterial reduction, regardless of whether it was applied for 3 or 1.5 minutes, which confirms experimental data generated with healthy volunteers.

Infect Control Hosp Epidemiol 2009; 30:420-426

Surgical hand antisepsis aims to minimize the release of skin bacteria into open wounds from the operating team members' hands, thereby helping to ensure optimal prevention of surgical site infection in the event of unnoticed glove perforation. ${ }^{1}$ Therefore, in contrast to hygienic hand washing or the use of hand rub, surgical hand antisepsis aims to eliminate all transient bacteria and to significantly reduce resident skin flora. The practice should, moreover, inhibit bacterial regrowth on gloved hands during surgery. ${ }^{2,3}$ Surgical hand antisepsis constitutes standard infection control practice all over the world, even though the actual impact of surgical hand antisepsis on the incidence of surgical site infection has not been investigated in randomized controlled clinical trials. ${ }^{4}$ The level of antimicrobial activity required to prevent surgical site infection is unknown, and standard efficacy levels for surgical hand antisepsis have therefore been established. European Norm (EN) 12791 defines a reference disinfection procedure. ${ }^{5}$ The efficacy of a product cleared for surgical hand antisepsis must, immediately after application and 3 hours thereafter, not be inferior to that of the reference procedure, which is defined as a 3 -minute hand rub with $60 \% \mathrm{vol} / \mathrm{vol}$ n-propanol.

Hand washing with medicated soap, most commonly soap containing chlorhexidine, had constituted routine practice for all healthcare personnel in the operating theater. More recently, several alcohol-based hand rubs have been licensed for commercial use in Europe. ${ }^{6-8}$ Compared with antimicrobial soaps, alcohol-based hand rubs have a broader antimicrobial spectrum, are faster acting and better tolerated, ${ }^{6,9-15}$ and save money by eliminating the need for warm water. ${ }^{16}$

From the Department of Surgery (W.P.W., S.R., D.O., W.R.M.), the Division of Infectious Diseases and Hospital Epidemiology (U.N., R.S., M.D., A.F.W.), and the Microbiology Laboratory (R.F.), University Hospital Basel, Basel, Switzerland; and the Department of Hygiene and Medical Microbiology, Medical University of Vienna, Vienna, Austria (M.L.R.).

Received July 31, 2008; accepted December 8, 2008; electronically published March 24, 2009.

(C) 2009 by The Society for Healthcare Epidemiology of America. All rights reserved. 0899-823X/2009/3005-0003\$15.00. DOI: 10.1086/596772 
Several studies have shown that scrubbing for 5 minutes reduces bacterial counts as effectively as scrubbing for 10 minutes. ${ }^{17-19}$ Other authors have suggested that scrubbing for 2 or 3 minutes reduces bacterial counts to acceptable levels. ${ }^{3,20-24}$ A 3-minute application of Sterillium classic pure (Bode Chemie), a propanol-based hand rub commonly used in Europe, has been shown not only to pass the test outlined in EN 12791 but also to be even more effective than the alcohol used in the reference procedure. ${ }^{25}$ Very recently, a 90second application of Sterillium classic pure was proven to be at least as effective as the 3-minute reference disinfection procedure for healthy volunteers under rigorous experimental conditions in the laboratory.

Since 1995, surgical hand antisepsis involving a 3-minute application of Sterillium classic pure has been the standard procedure at the University Hospital Basel (Basel, Switzerland). Shortening the disinfection time to 1.5 minutes without diminishing the required efficacy would offer obvious advantages. The present study aimed to validate the experimental findings described above under clinical conditions, to test the hypothesis that the antibacterial efficacy of 1.5- and 3-minute applications of this product was similar, both immediately after application and after surgery.

\section{METHODS}

\section{Product and Formulation}

Sterillium classic pure, the standard product used for surgical hand antisepsis at the University Hospital Basel, was used for all surgical hand hygiene in this study. Its main components are $45 \% \mathrm{vol} / \mathrm{vol} 2$-propanol, $30 \% \mathrm{vol} / \mathrm{vol} 1$-propanol, and $0.2 \%$ mecetronium ethylsulphate; the remaining components include purified water, 1-tetradecanol, and $85 \%$ glycerol.

\section{Setting}

The study was conducted mainly in the general surgery operating rooms of the University Hospital Basel from April through September 2007. The 32 participating surgeons, all volunteers, included the head of the division, attending physicians, fellows, and residents specializing in vascular, visceral, traumatological, urological, or gynecological surgery. To preclude possible distortion due to the reduction of microbial flora by performance of surgical hand antisepsis earlier in the day, the study was restricted to participating surgeons' first operation of the day. No exclusion criteria were applied, to ensure that the study was conducted under real-world conditions. Consequently, any surgeon who felt capable of operating was qualified to participate in the study. Surgeons were instructed to apply hand rub for 1.5 or 3 minutes; assignments were randomly allocated by a computer-generated list. The study was approved by the human subjects com- mittee as part of the quality improvement program of the University of Basel.

\section{Testing Principles and Prerequisites}

In principle, the study design adhered to the methods outlined in the EN 12791 for surgical hand antisepsis. ${ }^{26}$ In brief, a formulation for surgical hand disinfection is compared with a standardized 3-minute reference hand rub procedure with a hand rub that includes $60 \% \mathrm{n}$-propanol vol/vol. The formulation being tested must not be statistically significantly less effective than the reference treatment. The procedure requires parallel testing on the hands (up to the wrists) of 18-20 volunteers, who-in accordance with a randomized, crossover design-perform both procedures in 2 phases separated by an interval of 1 week.

Because these guidelines were not primarily designed for in-use clinical evaluation, a few modifications were deemed necessary to conduct the study in a clinical setting. First, no limitation was placed on the use of substances that might affect normal microbial populations on the skin, such as deodorants, shampoos, lotions, or soaps. Second, no additional care of the fingernails was required for participants. Third, the sustained effect was determined after the end of the surgical procedure rather than strictly after 3 hours of wearing gloves.

All participants were interviewed after each experiment with a questionnaire that addressed all the EN 12791 recommendations, ${ }^{27}$ with the aim of assessing the differences between the experimental design prescribed in the standard and the conditions of present study. The questionnaire collected information about each participating surgeon's relevant characteristics as well as the baseline variables for the surgical procedures. Before each experiment, a baseline photograph was taken of both sides of both the participant's hands with a 4.0-megapixel digital camera (PowerShot A80; Canon) to evaluate the possible adverse effects of the test product on surgeons' skin.

\section{Assessment of Baseline Values in the Operating Theater}

Surgeons rubbed the distal phalanges of their right and left hands for 1 minute against the bottom of a sterile metal bowl $(8 \mathrm{~cm}$ in diameter) that contained $10 \mathrm{~mL}$ of tryptic soy broth. We took $1.0-\mathrm{mL}$ and $0.1-\mathrm{mL}$ aliquots of the sampling fluid from each hand as well as a $0.1-\mathrm{mL}$ aliquot of a $10^{-1}$ dilution of the sampling fluid and transferred them to trypticase agar plates. No more than 60 minutes elapsed between sampling and inoculation of the trypticase agar plates, which were incubated for a total of 48 hours at $35^{\circ} \mathrm{C} \pm 11^{\circ} \mathrm{C}$ in accordance with standard microbiological procedures.

\section{Disinfection Phase}

As previously outlined, surgeons were randomly allocated to the 1.5-minute or 3-minute group; hand rub was applied 
under direct supervision of the research nurse. All surgeons ultimately performed both experiments, each thereby acting as his or her own control. Surgeons were instructed and observed to keep their hands wet during the entire surgical hand antisepsis procedure, a requirement that has been shown to be more important than the volume of hand rub used. ${ }^{28}$ The investigators ensured that all 6 steps of the EN 12791 handrubbing technique ${ }^{27}$ were followed by all surgeons-especially with regard to fingertips, nail folds, and interdigital spaces. In addition to the oral instructions provided, a poster explaining the method was placed on the wall over the scrub sink. All surgeons rubbed their forearms as part of the disinfection process, as recommended for clinical practice by various national guidelines. ${ }^{4,8}$ The exact disinfection time was monitored with a stopwatch. At least 1 week elapsed between the 2 phases of the experiment to ensure reconstitution of normal skin flora.

\section{Assessment of Bacterial Counts After Application}

After the surgeons' hands were completely dry, they rubbed their fingers in tryptic soy broth for 1 minute-as they did prior to disinfection-to evaluate the immediate effect of the product. Thereafter, they dried their hands with a sterile paper towel before donning surgical gloves. Immediately after surgery, the surgeons' sterile coat and gloves were removed by a member of the research team, and a final sample was taken to determine the sustained effect of the disinfection procedure. Finally, all gloves were filled with water to examine each finger separately for leaks.

\section{Microbiologic Analysis}

Separate quantitative cultures were performed using $0.1 \mathrm{~mL}$ of the sampling fluids obtained from each hand. The following dilutions in phosphate buffered saline were used for the 3 -step assessment: undiluted, $1: 10,1: 100$, and $1: 1,000$ before disinfection; undiluted after disinfection; undiluted and $1: 10$ after surgery. The aliquots were transferred to trypticase agar plates and spread evenly over the surface with a sterile glass spatula. No more than 1 hour was allowed to elapse between sampling and inoculation of the trypticase agar plates. The plates were incubated for a total of 48 hours at $35^{\circ} \mathrm{C} \pm 1^{\circ} \mathrm{C}$, and the count of colony-forming units on each plate was assessed on a scale of 1-99. Then, these numbers were multiplied by the relevant dilution factor. Any bacterial species that were not normal skin flora were recorded separately. Coagulase-negative staphylococci, $\alpha$ and nonhemolytic streptococci, coryneform rods, and other gram-positive rods were classified as skin flora. The results were recorded as colony-forming units per milliliter in a spreadsheet and listed on a logarithmic scale as $\log _{10}$ colony-forming units. All microbiological examinations were performed by a designated research laboratory technologist. A mixture of $3.0 \%$ Tween $80,0.3 \%$ lecithin, $0.1 \%$ L-histidine hydrochloride, and
$3.0 \%$ saponin was evaluated for its disinfectant-neutralizing efficacy in multiple experiments to optimize the bacterial yield.

\section{Statistical Analysis}

All predisinfection and postdisinfection values were expressed in terms of $\log _{10}$. For the purposes of calculation, plate count values of 0 were reset to 1 because the $\log _{10}$ of 0 is undefined and the $\log _{10}$ of 1 is 0 . The immediate effect was calculated as the difference between baseline values and the values after application expressed as the logarithmic reduction factor. The sustained effect was determined by calculating the difference between the value obtained immediately after application and the value obtained after completing the surgical intervention and removing the surgical gloves.

The sample size-outlined in EN $12791^{27}$-was extended from 18 subjects to $20-32$ subjects to increase power and allow for individuals who dropped out of the study. Sample size calculations prohibited any inference about the incidence of surgical site infection, ${ }^{13}$ and therefore, such data were not collected during the study period.

Statistical analysis was performed by use of the Wilcoxon matched-pair signed rank test, in accordance with the requirements of EN $12791 .{ }^{27}$ Additional statistical analyses were performed using analysis of variance and the $\chi^{2}$ test, as appropriate. Additional analyses were performed that went beyond the factors addressed in EN 12791 to adjust for confounding variables such as duration of surgery, glove changes during surgery, and routine use of double-gloving. Two-tailed tests were used, and a $P$ value less than .05 was considered statistically significant. Statistical analyses were conducted with SPSS, version 14.0 (SPSS).

\section{RESULTS}

\section{Surgeons and Surgical Procedures}

The University Hospital Basel employs 186 surgeons in 10 different specialties; 118 work in the 5 specialties that were included in the study. A total of 32 surgeons participated in the trial. Surgeons were excluded who went on vacation during the trial, as well as surgeons who were likely to be absent because of an international meeting or had other' reasons not to fully complete the trial. However, the main reason for excluding participants was the defined sample size, and resources did not allow the inclusion of all surgeons willing to participate. All 32 participating surgeons completed the trial. The demographic characteristics of the participating surgeons are outlined in Table 1 . The 2 groups were similar in terms of their use of double-gloving, the practice of changing gloves, and other variables (Table 2). The mean duration of surgery was longer in the 3-minute group, but the difference was not statistically significant (Table 2). Surprisingly, 7 surgeons 
TABLE 1. Characteristics of the 32 Surgeons Who Participated in a Study Comparing Different Application Times for Alcohol-Based Hand Gel

\begin{tabular}{|c|c|}
\hline Characteristic & Value \\
\hline Age, mean $\pm S D$, years & $36 \pm 6.1$ \\
\hline Female sex & 31.3 \\
\hline Professional experience, mean $\pm S D$, years & $9.3 \pm 5.8$ \\
\hline \multicolumn{2}{|l|}{ Job description } \\
\hline Head of division & $1(3.1)$ \\
\hline Attending physician & $5(15.6)$ \\
\hline Fellow & $11(34.4)$ \\
\hline Resident & $15(46.9)$ \\
\hline \multicolumn{2}{|l|}{ Speciality } \\
\hline Visceral surgery & $17(53.1)$ \\
\hline Traumatology & $2(6.3)$ \\
\hline Vascular surgery & $5(15.6)$ \\
\hline Urology & $5(15.6)$ \\
\hline Gynecology & $3(9.4)$ \\
\hline History of allergy & $9(28.1)$ \\
\hline \multicolumn{2}{|l|}{ Routine use of double-gloving } \\
\hline No & $17(53.1)$ \\
\hline Sometimes & $12(37.5)$ \\
\hline Yes & $3(9.4)$ \\
\hline \multicolumn{2}{|l|}{ Routinely changes gloves during surgery ${ }^{2}$} \\
\hline No & $1(3.1)$ \\
\hline Before implantation of foreign bodies & $16(50)$ \\
\hline In case of damaged gloves & $28(87.5)$ \\
\hline Use of antibiotics ${ }^{\mathrm{b}}$ & $4(12.5)$ \\
\hline \multicolumn{2}{|l|}{ Use of skin ointment on hands ${ }^{b}$} \\
\hline No & $10(31.3)$ \\
\hline Yes-containing cortisone & $2(6.3)$ \\
\hline Yes-other & $20(62.5)$ \\
\hline
\end{tabular}

$(10.9 \%)$ reported local injuries on the hands in the 2 weeks prior to study entry (Table 2). One surgeon had a thermal injury from cautery, 1 had damage to a fingernail, and others had minor injuries that were no longer visible in the digital photographs.

\section{Bactericidal Efficacy}

The $\log _{10}$ colony-forming unit counts obtained before (baseline) and after application of the product tested (immediate effect) as well as after surgery (sustained effect) for the 1.5and 3-minute groups are given in the Figure. The mean ( \pm SD) $\log _{10}$ reduction factors immediately after application were $2.66 \pm 1.13$ for the 1.5 -minute group and $3.01 \pm$ 1.06 for the 3 -minute group $(P=.204)$. Similarly, no statistically significant difference was observed between the 2 groups with respect to the sustained effect values, which were a mean $( \pm S D)$ increases of $1.08 \pm 1.13 \log _{10} \mathrm{cfu}$ in the 1.5minute group and $0.95 \pm 1.27 \log _{10}$ cfu in the 3 -minute group $(P=.708)$. In the multivariate analyses, there was no statistically significant association between bacterial density on the skin after disinfection and any of the variables that were evaluated during the study, such as duration of the surgical procedure, glove punctures, type of surgery performed, routine use of double-gloving, or glove changes during surgery (data not shown).

\section{Adverse Effects on the Skin}

All participants had been using the test product during their surgical hand antisepsis routine for months before the present study was undertaken. None of the participants reported adverse effects on the skin from use of the test product, nor were any such effects identified in the pictures taken for the present study. All surgeons continued to use the test product after completion of this study.

\section{DISCUSSION}

This prospective randomized trial provides strong evidence that the rubbing time for Sterillium classic pure can be shortened from 3 to 1.5 minutes. To our knowledge, this is the first such study conducted under clinical conditions in accordance with EN 12791 requirements. ${ }^{27}$ No statistically significant differences were observed in the immediate or sustained reduction of resident hand flora when surgeons applied the test product for 1.5 instead of 3 minutes.

The European test standard EN 12791 was developed in $1997 .^{27}$ To fulfill its requirements, any new test formulation must not be significantly less effective than the standardized reference treatment, which consists of $60 \% \mathrm{vol} / \mathrm{vol} 1$-propanol (n-propanol), applied for 3 minutes. A single, 3-minute application of the product tested in the present study has been shown to meet EN 12791 requirements. ${ }^{25}$ Kampf et al. ${ }^{7}$ showed that even a 1.5 -minute application of this product sufficed to meet these requirements in a group of 18-20 healthy volunteers. The Kampf et al. study, ${ }^{7}$ however, did not reflect the application of the test product under actual operating room conditions, because disinfection entailed rubbing both hands up to the wrists only. Moreover, the subjects' hands had healthy, unexceptional skin, and their nails were short and clean. All subjects washed their hands with an unmedicated soft soap (sapo kalinus, ie, potash soap) prior to surgical hand antisepsis to remove transient bacterial flora and any foreign particles. Volunteers used no substances on their hands or arms that were known to affect normal skin microbial populations for 1 week prior to testing. Finally, a washout period of at least 1 week between phases of the experiment was established for the reconstitution of normal skin flora. Hence, the Kampf et al. study did not explore whether disinfection with the test product for 1.5 minutes led to a sufficient reduction of resident hand flora in routine clinical practice.

In the present study, all surgeons who performed surgery during the test period were eligible to participate. All surgeons 
included their forearms in the disinfection process, which is not part of the EN 12791 requirements but is in accordance with recommendations made elsewhere. ${ }^{4,8}$ This part of the procedure took approximately 15-30 seconds, but total exposure time was difficult to assess because surgeons poured the alcohol-based hand rub onto their palms. Nonetheless, rubbing the forearms could conceivably reduce the effectiveness of the agent on the hands. In fact, Kampf et al. ${ }^{7}$ showed that the use of $2-3 \mathrm{~mL}$ of the test product for 1 minute was significantly less effective than the 3-minute reference procedure called for in EN 12791. Hands and forearms should be kept wet during the whole disinfection procedure to ensure maximum effect, so the necessary volume of hand rub is determined by the size of the person's hands and forearms. There were $21(65.6 \%)$ surgeons in the 1.5-minute group and $24(75 \%)$ in the 3 -minute group who were reported not to have washed their hands before entering the operating room, although such washing is required by institutional guidelines. Moreover, $22(68.8 \%)$ of the participants had used substances on their hands and arms during the 3 months prior to testing that might have affected normal microbial populations on the skin. Finally, most surgeons performed surgical hand antisepsis for surgical procedures not monitored in this study on a number of occasions during the week between the 2 phases of this experiment.

Despite these apparent deviations from the protocol adapted to EN 12791, the 1.5- and 3-minute application periods proved to be similarly effective for both immediate and sustained bacterial reduction. Nonetheless, not all alcoholbased hand rubs have been shown to be EN 12791-compliant in in vivo experiments, and shortened rubbing times should only be considered for products that have been cleared for 1.5-minute application relative to the standard, preferably supported by trials such as the one reported here. Furthermore, the trend towards slightly greater effectiveness observed

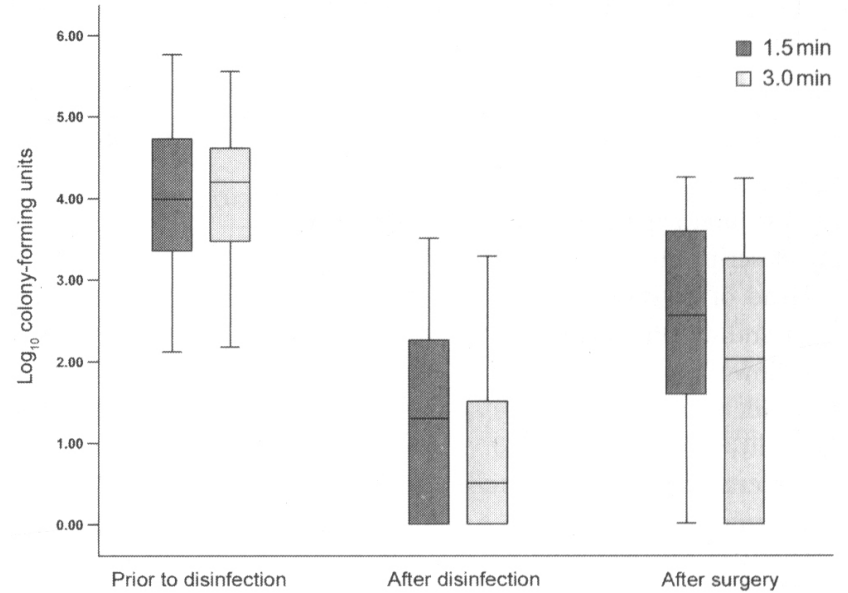

FIGURE Colony-forming unit (cfu) counts obtained before application (baseline), after application of alcohol-based hand rub for either 1.5 or 3 minutes (immediate effect), and after surgery (sustained effect). The mean ( \pm SD) values for the 1.5-minute group were $3.98 \pm 0.96 \log _{10}$ cfu before disinfection, $1.32 \pm 1.15 \log _{10}$ cfu after disinfection, and $2.41 \pm 1.31 \log _{10}$ cfu after surgery; for the 3 minute group, these same values were $3.93 \pm 1.10 \log _{10} \mathrm{cfu}$, $0.92 \pm 1.06 \log _{10} \mathrm{cfu}$, and $1.88 \pm 1.51 \log _{10} \mathrm{cfu}$.

for 3-minute application is an indication that 1.5 minutes is the shortest acceptable time for this formulation.

This study is subject to a number of limitations. First, surgeons were instructed before surgical hand antisepsis and continuously observed during the procedure. The technique used to apply hand rub is crucial to its effectiveness, so technique was continuously observed during the study because the study design did not focus on comparing different techniques. Therefore, the safety margin may go below the ref-

TABLE 2. Characteristics of Surgical Procedures Performed by 32 Surgeons, According to Duration of Surgical Hand Antisepsis

\begin{tabular}{|c|c|c|c|}
\hline \multirow[b]{2}{*}{ Characteristics of procedures } & \multicolumn{2}{|c|}{ Duration of antisepsis } & \multirow[b]{2}{*}{$P$} \\
\hline & $1.5 \mathrm{~min}$ & $3 \mathrm{~min}$ & \\
\hline No. of procedures & 32 & 32 & \\
\hline Duration of surgery, mean $( \pm S D)$, min & $82.9 \pm 50.3$ & $105.3 \pm 78.2$ & .18 \\
\hline Surgeon washed hands with medicated soap before entering the operating room area & $11(34.4)$ & $8(25)$ & .58 \\
\hline Surgeon changed gloves during surgery & & & .8 \\
\hline Once & $7(21.9)$ & $5(15.6)$ & \\
\hline More than once & $0(0)$ & $1(3.1)$ & \\
\hline Never & $25(78.1)$ & $26(81.3)$ & \\
\hline Surgeon used double-gloving during surgery & $3(9.4)$ & $5(15.6)$ & .70 \\
\hline Surgeon's gloves were perforated during surgery ${ }^{a}$ & $3(9.4)$ & $9(28.1)$ & .10 \\
\hline Surgeon reported injuries on hands ${ }^{\mathrm{b}}$ & $2(6.3)$ & $5(15.6)$ & .26 \\
\hline
\end{tabular}

NOTE. Data are no. (\%) of surgeons, unless otherwise indicated.

"If double-gloving was used, this refers to tiny holes in the inner glove.

b Within 2 weeks before surgery. 
erence standard if surgeons do not strictly follow the technique that is outlined in EN $12791^{27}$ and supported by the World Health Organization guideline. Second, neither the type nor the duration of surgery was standardized, although surgeons were randomly allocated to the 1.5- or 3-minute group. Differences in several baseline variables, such as duration of surgery, glove perforations, and hand injuries, might have influenced the level of antimicrobial efficacy. However, several multivariate analyses were unable to detect any statistically significant confounder. Third, the sample size precluded identification of any association with the incidence of surgical site infection, the ultimate outcome variable in such studies. No statistically significant differences were observed, however, in a trial involving over 2,000 patients that compared surgical hand antisepsis performed with medicated soap to antisepsis performed with an alcohol-based hand rub, both applied for 5 minutes. ${ }^{13}$ Finally, there were differences between the 2 groups, such as the duration of surgery. Even though the differences were not statistically significant, the sample size would not allow for adjustments for multiple confounding variables.

In conclusion, this strictly clinical trial confirms the findings of an in vivo experimental study conducted by Kampf et al., ${ }^{7}$ who reported that 1.5 minutes of surgical hand antisepsis with the alcohol-based compound tested in the present study is an EN 12791-compliant procedure. The results of the present study provide evidence that supports shortening the time of surgical hand antisepsis with the compound tested to 1.5 minutes. The potential time savings could prove to be clinically relevant, especially for short briefings of the operating team prior to incision and in emergency situations.

\section{ACKNOWLEDGMENTS}

Potential conflicts of interest. All authors report no conflicts of interest relevant to this work.

Financial support. This research was funded by the University of Basel and an unrestricted grant from Bode Chemie. The sponsors had no role in study design or implementation, data collection, management, analysis or interpretation, or preparation, review, or approval of the manuscript.

Address reprint requests to Andreas F. Widmer, Division of Infectious Diseases and Hospital Epidemiology, University Hospital Basel, Petersgraben 4, CH-4031 Basel, Switzerland (awidmer@uhbs.ch).

\section{REFERENCES}

1. Kampf G, Goroncy-Bermes P, Fraise A, Rotter M. Terminology in surgical hand disinfection-a new Tower of Babel in infection control. J Hosp Infect 2005; 59:269-271.

2. Trampuz A, Widmer AF. Hand hygiene: a frequently missed lifesav- ing opportunity during patient care. Mayo Clin Proc 2004; 79:109116.

3. Widmer AF. Replace hand washing with use of a waterless alcohol hand rub? Clin Infect Dis 2000; 31:136-143.

4. Boyce JM, Pittet D. Guideline for hand hygiene in health-care settings: recommendations of the Healthcare Infection Control Practices Advisory Committee and the HICPAC/SHEA/APIC/IDSA Hand Hygiene Task Force. Society for Healthcare Epidemiology of America/Association for Professionals in Infection Control/Infectious Diseases Society of America. MMWR Recomm Rep 2002; 51:1-45.

5. Rotter M, Kundi M, Suchomel M, et al. Reproducibility and workability of the European test standard EN 12791 regarding the effectiveness of surgical hand antiseptics: a randomized, multicenter trial. Infect Control Hosp Epidemiol 2006; 27:935-939.

6. Kampf G, Hollingsworth A. Validity of the four European test strains of prEN 12054 for the determination of comprehensive bactericidal activity of an alcohol-based hand rub. J Hosp Infect 2003; 55:226231.

7. Kampf G, Ostermeyer C, Heeg P. Surgical hand disinfection with a propanol-based hand rub: equivalence of shorter application times. $J$ Hosp Infect 2005; 59:304-310.

8. Labadie JC, Kampf G, Lejeune B, et al. Recommendations for surgical hand disinfection-requirements, implementation and need for research: a proposal by representatives of the SFHH, DGHM and DGKH for a European discussion. I Hosp Infect 2002; 51:312-315.

9. Kampf G, Rudolf M, Labadie JC, Barrett SP. Spectrum of antimicrobial activity and user acceptability of the hand disinfectant agent Sterillium Gel. J Hosp Infect 2002; 52:141-147.

10. Kampf G, Muscatiello M, Hantschel D, Rudolf M. Dermal tolerance and effect on skin hydration of a new ethanol-based hand gel. J Hosp Infect 2002; 52:297-301.

11. Kampf G, Muscatiello M. Dermal tolerance of Sterillium, a propanolbased hand rub. J Hosp Infect 2003; 55:295-298.

12. Kampf G, Kramer A. Epidemiologic background of hand hygiene and evaluation of the most important agents for scrubs and rubs. Clin Microbiol Rev 2004; 17:863-893.

13. Parienti JJ, Thibon P, Heller R, et al. Hand-rubbing with an aqueous alcoholic solution vs. traditional surgical hand-scrubbing and 30day surgical site infection rates: a randomized equivalence study. JAMA 2002; 288:722-727.

14. Pietsch H. Hand antiseptics: rubs versus scrubs, alcoholic solutions versus alcoholic gels. J Hosp Infect 2001; 48(Suppl A):S33-S36.

15. Rotter ML. Arguments for alcoholic hand disinfection. J Hosp Infect 2001; 48(Suppl A):S4-S8.

16. Jehle K, Jarrett N, Matthews S. Clean and green: saving water in the operating theatre. Ann R Coll Surg Engl 2008; 90:22-24.

17. Dineen P. An evaluation of the duration of the surgical scrub. Surg Gynecol Obstet 1969; 129:1181-1184.

18. Galle PC, Homesley HD, Rhyne AL. Reassessment of the surgical scrub. Surg Gynecol Obstet 1978; 147:215-218.

19. O'Farrell DA, Kenny G, O'Sullivan M, Nicholson P, Stephens M, Hone R. Evaluation of the optimal hand-scrub duration prior to total hip arthroplasty. I Hosp Infect 1994; 26:93-98.

20. Hingst V, Juditzki I, Heeg P, Sonntag HG. Evaluation of the efficacy of surgical hand disinfection following a reduced application time of 3 instead of $5 \mathrm{~min}$. J Hosp Infect 1992; 20:79-86.

21. Lowbury EJ, Lilly HA. Disinfection of the hands of surgeons and nurses. Br Med J 1960; 1:1445-1450.

22. O'Shaughnessy M, O'Malley VP, Corbett G, Given HF. Optimum duration of surgical scrub-time. Br J Surg 1991; 78:685-686.

23. Pereira LJ, Lee GM, Wade KJ. The effect of surgical handwashing routines on the microbial counts of operating room nurses. Am J Infect Control 1990; 18:354-364.

24. Wheelock SM, Lookinland S. Effect of surgical hand scrub time on subsequent bacterial growth. AORN J 1997; 65:1087-1092.

25. Marchetti MG, Kampf G, Finzi G, Salvatorelli G. Evaluation of the 
bactericidal effect of five products for surgical hand disinfection according to prEN 12054 and prEN 12791. J Hosp Infect 2003; 54: 63-67.

26. Rotter ML. European norms in hand hygiene. J Hosp Infect 2004; 56(Suppl 2):S6-S9.

27. European Committee for Standardization. EN 12791. Chemical dis- infectants and antiseptics- surgical hand disinfection-test method and requirements (phase 2/step 2). Brussels: European Committee for Standardization; 1997.

28. Kampf G, Ostermeyer C. Influence of applied volume on efficacy of 3-minute surgical reference disinfection method prEN 12791. Appl Environ Microbiol 2004; 70:7066-7069. 